\title{
Quelques remarques préliminaires
}

Centrées sur ses manifestations en langue et en discours (... et en parole, voudront assurément compléter les phonéticiens), les contributions ici présentées prennent pour objet d'étude général la perception humaine, mais qu'elles abordent exclusivement sous ses coutures linguistiques; les domaines investis étant principalement, en effet, ceux de la lexicologie (ou lexicographie), de la morphologie, de la syntaxe, de la sémantique, de la phonétique (y compris pathologique) et de l'énonciation. Pour ce qui est des approches adoptées, par ailleurs, ce sont les options descriptive (Brigitte Garcia \& Emmanuella Martinod ; Aude Grezka ; Iris Eshkol-Taravella \& Hélène Flamein ; Alain Rabatel), historique (Maria Aldea), contrastive (Stéphanie Béligon ; Ivana Didirková, Agnès Steuckardt \& Fabrice Hirsch ; Christelle Lacassain-Lagoin) et didactique (Dominique Hamm \& Magdalena Dańko) qui l'emportent. Mais pourquoi consacrer ainsi des travaux de Sciences du langage à la perception considérée du point de vue linguistique ?

D'abord, l'Homme se trouvant de fait à la jonction d'au moins cinq sens, que sont la vue, l'ouïe, l'odorat, le goût et le toucher, et dont il est pour ainsi dire le greffier naturellement désigné, les linguistes que nous sommes ont jugé opportun de s'interroger sur la manière dont le langage naturel prenait en charge les perceptions (tant en termes d'encodage lexical, phrastique ou sémiotique, d'architecture syntaxique, de configuration sémantique, de point de vue, de production, de transmission que de réception) pour tenter de rendre plus nets les contours encore flous de l'expression linguistique de la perception, d'en approfondir l'étude des propriétés ou spécificités connues, voire d'en mettre au jour de nouvelles. Par ailleurs, force nous a été de constater que malgré l'augmentation continue du nombre d'études linguistiques relatives à la perception depuis le milieu du XXe siècle, aujourd'hui, paradoxalement, celle-ci ne parvient toujours pas, dans nos ouvrages grammaticaux quels qu'ils soient (grammaires, précis, dictionnaires, terminologies ou référentiels), à trouver sa place comme contenu à part entière (ni comme chapitre ni comme section, pas même comme sous-section) ; alors que dans le même temps, les manuels scolaires, quant à eux, y compris ceux de Français Langue Étrangère, semblent mettre un point d'honneur à toujours proposer au moins une rubrique destinée à montrer quelles constructions-types permettent de rendre compte de quels types de perceptions.

C'est Maria Aldea ( ${ }^{1}$ L'expression de la perception dans le Lexicon de Buda (1825) », pp. 11-28) qui ouvre les débats, avec une étude portant sur les diverses manières d'exprimer la perception dans un dictionnaire roumain ancien, connu dans la littérature de spécialité sous le nom de Lexicon de Buda (réalisé par un groupe d'érudits transylvains, ce dictionnaire représente en effet une époque charnière dans la lexicographie roumaine moderne). En pratique, l'auteur fait d'abord l'inventaire des mots de la perception en se servant, pour leur sélection, et des équivalents latins disponibles et de l'analyse sémique, tout en se rapportant aux dictionnaires roumains contemporains; ensuite, elle tâche de mettre en évidence à la fois certains

\footnotetext{
${ }^{1}$ Université Babeş-Bolyai, Cluj, Roumanie
} 
aspects normatifs anciens par rapport aux normes actuelles, l'évolution sémantique des mots (si mentionnée) et le traitement lexicographique de ceux-ci - mais sans prendre en considération d'autres approches, fussent-elles contrastives ou ethnolinguistiques. Finalement, c'est une perspective plus élargie qui se dessine sur la circulation et l'évolution en diachronie de ces mots de la perception dans une aire géographique où la langue roumaine ne bénéficiait pas, à l'époque, d'une reconnaissance officielle.

Les études suivantes orientent les débats vers des points d'analyse plus resserrés, la première d'entre elles, menée par Stéphanie Béligon ${ }^{2}$ ( « Feel et feeling : tel verbe, tels noms?», pp. 29-45), s'intéressant au verbe feel et à ses correspondants (étymologiques et morphologiques) nominaux du point de vue sémantique. Le verbe, qui renvoie à la perception, à la sensation, ainsi qu'à la cognition, admet au moins deux constructions : s'il a souvent pour sujet syntaxique le percevant (ou expérient) de la sensation, du sentiment ou de l'émotion (I was feeling guilty), parfois, en effet, ce peut être un objet qui suscite cette perception ou impression constituant son sujet grammatical (This wallet feels like leather). Et, sémantiquement parlant, à ces deux constructions verbales semblent bien correspondre respectivement les deux substantifs feel et feeling, le premier constituant une nominalisation du verbe par conversion et paraissant plutôt désigner ce qui a trait à ce qui est perçu, le second en constituant une nominalisation par suffixation en -ing et paraissant plutôt désigner ce que ressent l'expérient. Pourtant, si les deux lexèmes se distinguent d'ordinaire, ils se rejoignent malgré tout dans certaines de leurs significations, tous deux pouvant renvoyer, par exemple, à une ambiance ou une atmosphère (They have managed to recreate the feeling of the original theatre; It's a big city but it has the feel of a small town); et c'est bien là le cœur de cette contribution, le double objectif avoué de l'auteur étant de déterminer jusqu'à quel point ces deux lexèmes se ressemblent quand ils sont apparemment synonymes mais de démontrer aussi qu'ils présentent le perçu de façon distincte. En pratique, après avoir comparé les significations du verbe feel et des substantifs feel et feeling, l'auteur examine des données recueillies dans le COCA (Corpus of Contemporary American English) concernant l'emploi des deux noms avant que d'évoquer, in fine, quelques-unes des particularités de la modalité du sentir dite par le verbe feel pour rendre compte de cette jonction entre les noms feel et feeling.

Après l'expression de la perception, la perception de l'expression : l'étude conduite par Ivana Didirková ${ }^{3}$, Agnès Steuckardt ${ }^{4}$ et Fabrice Hirsch ${ }^{5}$ (« La classe grammaticale a-t-elle une influence dans l'apparition des disfluences perçues comme sévères ? Une étude sur le français et le slovaque », pp. 47-60) s'intéresse au bégaiement, en effet. La production orale d'énoncés se fait rarement sans quelques ruptures de la fluence. Ces accidents de parole, également appelés disfluences, passent généralement inaperçus qui indiquent simplement que les capacités du locuteur à émettre un message sont momentanément inférieures aux

${ }^{2}$ Université Paris-Est, Marne-la-Vallée (LISAA, EA 4120), France

${ }^{3}$ F.R.S.-FNRS \& Université catholique de Louvain, Belgique

${ }^{4}$ UMR 5267 Praxiling \& CNRS, Université Paul-Valéry Montpellier 3, France

${ }^{5}$ UMR 5267 Praxiling \& CNRS, Université Paul-Valéry Montpellier 3, France 
exigences linguistiques requises pour ce faire ; toutefois, il arrive qu'ils soient (bien) davantage apparents, comme dans certains troubles de la parole, tel le bégaiement, altération de la fluence de la parole ayant des conséquences négatives sur la communication et se caractérisant par la présence d'accidents de parole sévères empêchant la personne qui en souffre de s'exprimer de manière fluide. Or, si la plupart des études actuelles concluent que la localisation des disfluences dépend avant tout de facteurs moteurs, quelques chercheurs ont pourtant établi un lien entre le risque de voir apparaître une disfluence et d'autres niveaux linguistiques : il a notamment été montré, ainsi, que les mots lexicaux étaient plus bégayés que les grammaticaux, que les personnes qui bégaient produisaient davantage de disfluences sur les verbes que sur les substantifs ou que les substantifs constituaient, en parole conversationnelle, la classe grammaticale la moins disfluente parmi tous les mots lexicaux. Aussi les auteurs de cette étude contrastive entre le français et le slovaque se sont-ils fixé comme objectif de vérifier s'il existait effectivement des régularités dans les structures linguistiques touchées par les bégayages, et lesquelles exactement.

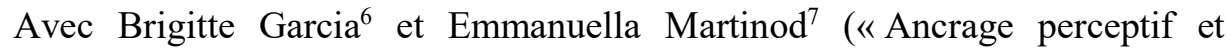
invariant dans les langues des signes (LS), langues de sourds », pp. 73-88), ce sont des langues naturelles d'un (tout) autre type qui sont mises à l'honneur, en l'occurrence celles des signes. Ces langues visuo-gestuelles, pratiquées par les sourds à travers le monde, sont caractérisées, d'une part, par une forte iconicité, flagrante à tous les niveaux de l'analyse linguistique, et semblent présenter entre elles, d'autre part, un degré d'invariant bien plus élevé que ce qu'il en est entre les langues de modalité audio-phonatoire, dites vocales, pratiquées par la majorité entendante. Aussi la présente contribution explore-t-elle l'hypothèse selon laquelle ce haut degré de similitude observé entre les LS du monde serait en lien étroit avec leur ancrage commun dans une iconicisation de l'expérience perceptivo-pratique humaine, directement structurante pour ces langues. Formellement, l'étude repose sur l'examen, en transversal d'un échantillon conséquent de LS, de l'un des composants minimaux de ces langues, la configuration manuelle.

À travers le lexique de la perception, Aude Grezka (« Morfetik, un dictionnaire morphologique : illustration avec le lexique de la perception », pp. 89-99) a choisi de présenter, quant à elle, un nouveau dictionnaire des mots simples du français, ressource morphologique appelée Morfetik et dont le système de traitement associe un moteur de flexion, un dictionnaire des formes fléchies, des interfaces de consultation et d'interrogation, ainsi qu'un ensemble d'outils permettant la maintenance et l'exploitation des ressources. Il est vrai que de nombreux chercheurs tentent actuellement de concevoir (et d'établir) de nouvelles méthodologies appropriées au traitement automatique des langues: à terme, il s'agit plus précisément de parvenir à modéliser une certaine partie de la connaissance

${ }^{6}$ UMR 7023 Structures Formelles du Langage, Université Paris 8-Université Paris Lumières \& CNRS, France

${ }^{7}$ UMR 7023 Structures Formelles du Langage, Université Paris 8-Université Paris Lumières \& CNRS, France

${ }^{8}$ Université Paris 13, Sorbonne Paris Cité \& Laboratoire Lexiques Dictionnaires Informatique (LDICNRS), France 
linguistique afin de la rendre exploitable par la machine, de créer de la ressource linguistique qui sera employée dans des applications informatiques (aide à la rédaction, à la traduction, etc.). Or, dans ce cadre, les systèmes de traitement automatique des langues (TAL) intègrent de plus en plus des connaissances de nature linguistique, parmi lesquelles la morphologie est très souvent sollicitée, en particulier dans le traitement des langues comme le français, à morphologie flexionnelle riche mais surtout complexe. Sachant que Morfetik, comme le souligne l'auteur in fine, n'est que l'un des éléments d'un système plus large impliquant un corpus continu et un module de néologismes, ainsi que différents outils pour suivre la fréquence d'usage des lexies du dictionnaire.

Comme le titre de leur contribution le laisse entendre («Perception et traque de «l'accent étranger»: la production des voyelles orales en FLE par des polonophones », pp. 101-122), Dominique $\mathrm{Hamm}^{9}$ et Magdalena Dańko ${ }^{10}$ s'intéressent, dans le cadre de la didactique de l'oral en FLE, à une phonétique contrastive particulière, dite corrective. Pour les auteurs, le constat est amer : ce type de phonétique constitue le parent pauvre du FLE depuis la première génération des approches communicatives, qui excluait toute référence à la norme prescriptive ; les enseignants manquent de formation, même si, actuellement, un retour en force de la phonétique s'opère et qu'une place, bien légitime, dans les séquences de manuels relevant de l'approche actionnelle lui est enfin accordée aux côtés des objectifs lexicaux, grammaticaux et socioculturels; les leçons restent traditionnelles et les exercices redondants, surtout relatifs l'articulatoire à l'aide de coupes sagittales ou à la perception par reconnaissance/discrimination à l'aide de paires minimales. Ainsi, après avoir dûment rappelé qu'un bilingue n'était pas un double monolingue (le but de tout apprentissage linguistique n'étant pas l'assimilation totale à une langue et à une culture étrangères, mais l'intégration d'une nouvelle langue et d'une nouvelle culture dans des ressources déjà présentes) et que ce qu'on appelait «l'accent étranger » ne dépendait pas du tout du fait que l'étranger en question ne pouvait pas prononcer un certain son, mais plutôt du fait qu'il n'appréciait pas correctement ce son (cette fausse appréciation des sons d'une langue étrangère étant conditionnée par la différence existant entre la structure phonologique de la langue étrangère et celle de la langue maternelle du sujet parlant), les auteurs tentent de montrer comment le type de remédiation didactique et pédagogique qu'elles prônent, fondé sur la méthode dite verbo-tonale d'intégration phonétique, est susceptible d'aider les apprenants à cheminer dans une atmosphère sonore nouvelle et leur faire obtenir une prononciation quasi authentique et inconsciente en éduquant leur audition (sachant que la séquence rythmico-intonative est précisément le moule dans lequel se réalise le phonème).

Quant à l'objectif de l'étude proposée par Iris Eshkol-Taravella ${ }^{11}$ et Hélène Flamein ${ }^{12}$ (« «Dis-moi Orléans ». Repérage et analyse de la perception d'un lieu dans l'oral transcrit », pp. 61-71), il est de présenter une analyse, effectuée dans le cadre du Traitement Automatique du Langage (TAL), de la perception des lieux par

\footnotetext{
${ }^{9}$ Université de Strasbourg, France

${ }^{10}$ Université d'Opole, Pologne

${ }^{11}$ Laboratoire Ligérien de Linguistique (UMR7270), France

12 Laboratoire Ligérien de Linguistique (UMR7270), France
} 
différents locuteurs dans le discours transcrit de l'oral, les techniques ainsi employées permettant de détecter automatiquement dans les transcriptions les lieux ainsi que les expressions qui les accompagnent. Pourquoi ? Il est vrai que la dénomination d'un lieu est un processus social réapproprié subjectivement et qu'elle est déterminée par la personnalité, l'histoire du locuteur ; un lieu peut être approprié, apprécié ou non par un locuteur; la perception d'un lieu peut aussi apparaître à travers l'avis, le jugement, le sentiment, la sensation, etc., exprimés par un locuteur et à travers sa dénomination. Repérer automatiquement un lieu et sa perception est donc une tâche difficile. Le corpus traité à cet effet, composé d'une vingtaine de modules représentant chacun des situations de communication différentes, pour un total de 460 heures d'enregistrement, est le corpus ESLO2 (Enquête SocioLinguistique à Orléans). Formellement, la contribution développe les principales étapes de ce travail de recherche: d'abord sont exposés les cadres théoriques utilisés, les auteurs n'omettant pas de rappeler les principes de la détection automatique des différentes désignations des lieux dans le discours; ensuite est explicitée la méthodologie, avec les différentes étapes de traitement, avant, enfin, que ne soit proposée une analyse des résultats obtenus.

Dans l'avant-dernier texte, de Christelle Lacassain-Lagoin ${ }^{13}$ («Voir et see: étude comparée de la construction à attribut de l'objet », pp. 123-146), l'objectif est cette fois-ci de comparer, dans une perspective énonciativiste et cognitiviste, les verbes de perception visuelle see en anglais (langue germanique) et voir en français (langue romane) dans des constructions où l'on dit traditionnellement que le complément d'objet direct du verbe est suivi d'un attribut. L'analyse, qui se concentre sur les attributs adjectivaux, nominaux et prépositionnels, tente plus précisément d'apporter réponse à deux questions principales: en lien avec le concept de coprédication, peut-on considérer que l'attribut de l'objet constitue une idée seconde par rapport à une idée première ?; par ailleurs, quels sont les liens entre syntaxe et sémantique dans les constructions à attribut de l'objet ? Et c'est ainsi que l'auteur s'interroge sur la connexion ou déconnexion entre syntaxe et sémantique dans les énoncés comportant une construction à attribut de l'objet, afin de déterminer s'il existe une relation entre la catégorie grammaticale de l'attribut et le sens véhiculé par l'énoncé. En pratique, l'étude se déroule en quatre temps : d'abord sont posées les particularités de see et voir et justifié le choix de leur traitement; sont présentées, ensuite, les caractéristiques des constructions à attribut de l'objet, ainsi que leurs similitudes et différences avec les structures transitives "simples" ; puis, l'auteur procède à une comparaison des trois types d'attributs de l'objet, notamment de leur distribution et des nuances sémantiques que chacun apporte à l'énoncé ; finalement, une synthèse est proposée relativement aux trois configurations étudiées et leurs emplois avec les deux verbes de perception en question. Précisons, également, que l'analyse repose sur des énoncés authentiques extraits de cinq corpus différents.

\footnotetext{
${ }^{13}$ Université de Pau et des Pays de l'Adour, Centre de Recherche en Poétique, Histoire Littéraire et Linguistique (CRPHLL), EA 3003, France
} 
Et c'est Alain Rabatel ${ }^{14}$ (« Pour une analyse énonciative et textuelle des points de vue perceptifs emphatiques (hétéro-perception et prise en charge énonciative) », pp. 147-172) qui refermera ce volume thématique sur la perception en langue et en discours. Alors qu'on distingue soigneusement les instances d'énonciation lorsque l'on procède à l'analyse des discours rapportés ou représentés, on ne fait pas de même, regrette l'auteur, lorsqu'il s'agit d'analyser des verbes de perception (VP), comme si l'origine énonciative n'avait guère d'importance. Or, on gagnerait à le faire, en distinguant le locuteur (L), source d'un acte de parole (ou d'énonciation) de l'énonciateur (E), instance source des points de vue, distinction qui permettrait, en effet, de rendre compte des cas de conjonction et de disjonction entre le locuteur/énonciateur premier (L1/E1) et le second (12/e2), en sorte qu'il ne faut pas confondre "l'énonciateur», qui est un parasynonyme du locuteur, avec l'énonciateur en tant qu'instance modale, c'est-à-dire la source à laquelle sont rapportés les choix de référenciation des objets du discours. Par ailleurs, la source énonciative, en tant que source modalisante d'un point de vue (spatio-temporel, cognitif, évaluatif, etc.), a des répercussions évidentes sur la référenciation et les valeurs modales des propositions qui contiennent des verbes de perception et leurs arguments, et, plus largement, sur l'ensemble des prédications, qui se présentent comme des apports du VP support, et sont susceptibles d'exprimer le monde perçu en fonction de la subjectivité, de l'intentionnalité du sujet centre de perspective, y compris dans le cas où ce dernier est un énonciateur second non locuteur. Ici, l'auteur se restreint à quelques verbes de perception, du type voir ou regarder, en confrontant ses analyses à d'autres relatives à ces mêmes verbes, même s'il reconnaît que d'autres choix auraient été possibles, concernant d'autres sens que la vue. D'une façon générale, il inscrit l'analyse des VP dans celle des comptes rendus de perception (CRP), en prenant en compte non seulement la phrase qui contient le VP, mais aussi les phrases subséquentes, parce que c'est souvent là que la perception se charge de valeurs intentionnelles et s'oriente vers l'action. Plus formellement, après avoir présenté rapidement la notion de point de vue dans le langage naturel puis en linguistique, ainsi que le cadre théorique de son analyse des perceptions, l'auteur examine les CRP avant que d'aborder dans un dernier point la question de leur prise en charge, par le locuteur/énonciateur premier et/ou second.

À titre de remarque liminaire finale, nous, les éditeurs de ce numéro thématique sur la perception, espérons que les divers points de vue linguistiques ici présentés donneront aux lecteurs un état des connaissances actualisé relativement aux différentes problématiques traitées. Il va sans dire, par ailleurs, que les auteurs comme les éditeurs apprécieraient tout retour constructif des lecteurs.

Elżbieta Biardzka, Magdalena Dańko, Greta Komur-Thilloy et Fabrice Marsac

${ }^{14}$ Université de Lyon 1, ICAR, France 